\title{
Research on the effect of cytokine concentration on the immune level and survival conditions of elderly patients with sepsis
}

\author{
XUGUANG LI and BIQING YAN \\ ICU, The Affiliated Hospital of Medical School, Ningbo University, Ningbo, Zhejiang 315000, P.R. China
}

Received October 13, 2017; Accepted May 17, 2018

DOI: $10.3892 / \mathrm{etm} .2018 .6221$

\begin{abstract}
The present study was carried out to investigate the effect of cytokine (CK) concentrations on the immune phenotype and survival conditions of elderly patients with sepsis. A total of 112 elderly patients with sepsis who were admitted to The Affiliated Hospital of Medical School, Ningbo University for treatment between June 2015 and June 2017 were enrolled as the subjects. According to each patient's condition, they were divided into the mild sepsis group $(n=68)$ and the severe sepsis group ( $\mathrm{n}=44)$. Additionally, a further 60 patients without sepsis who were admitted to the Intensive Care Unit (ICU) of this hospital during the same period were recruited as the control group. The levels of CK, tumor necrosis factor $\alpha$ (TNF- $\alpha$ ), interleukin (IL)-6, IL-8, procalcitonin (PCT), endothelin-1 (ET-1) and IL-10 were detected, and the assessment system for immune levels was established. The levels of TNF- $\alpha$, IL-6, IL-8, PCT, ET-1 and IL-10 in the serum of patients in the mild and severe sepsis groups were significantly higher than those in the control group $(\mathrm{P}<0.05)$, and the levels in the severe sepsis group were significantly higher than those in the mild sepsis group $(\mathrm{P}<0.05)$. Among the 112 sepsis patients, there were 30 mortalities $(26.79 \%)$ within 30 days, of which the levels of TNF- $\alpha$, IL-6, IL-8, PCT, ET-1 and IL-10 were significantly higher than those in the surviving patients $(\mathrm{P}<0.05)$. Between the mild and severe sepsis groups, as well as the number of mortalities and surviving patients, there were statistically significant differences when comparing the immunological phenotypes $(\mathrm{P}<0.05)$. These findings indicated that the levels of TNF- $\alpha$, IL-6, IL-8, PCT, ET-1 and IL-10 were associated with the condition and prognosis of elderly patients with sepsis, and the assessment system for immune levels based on the levels of these indicators was conducive to the stipulation of individualized immune regulation procedure and prognostic evaluation of sepsis.
\end{abstract}

Correspondence to: Dr Biqing Yan, ICU, The Affiliated Hospital of Medical School, Ningbo University, 247 Renmin Road, Ningbo, Zhejiang 315000, P.R. China

E-mail: bex9g6@163.com

Key words: sepsis, cytokine, disease condition, prognosis, immunological phenotype

\section{Introduction}

Sepsis is a kind of systemic inflammatory response syndrome (SIRS) caused by ischemia-reperfusion, severe trauma, shock, burn, infection and surgeries, and, essentially, is considered as the stress response of body to the infectious factors (1). Sepsis is believed to be the major cause accounting for septic shock, multiple organ failure (MOF) and multiple organ dysfunction syndrome (MODS) (2). Currently, the exact pathogenesis of sepsis remains unclear yet, but the immunological disorder is quite critical to the progression of sepsis. Cytokine (CK) is a kind of small proteins with extensive biological activities secreted by immunological cells or non-immunological cells, and, together with the complement and immune globulin, is called as the immune molecules, which are critical to the immune responses (3). Previous studies indicated that the level of CK and the relevant changes are closely correlated with the development, progression and prognosis of sepsis, and are considered as the potential indicators for monitoring the immunological function of patients with sepsis, and, thus, can be used for precise immunological regulation treatment (4). In this study, we enrolled the elder patients with sepsis who were admitted to this hospital for treatment between June 2015 and June 2017 as the subjects to monitor the changes in CK [tumor necrosis factor $\alpha$ (TNF- $\alpha$ ), interleukin-6 (IL-6), IL-8, procalcitonin (PCT), endothelin-1 (ET-1) and IL-10], so as to analyze the correlations between $\mathrm{CK}$ and prognosis of patients.

\section{Materials and methods}

Clinical materials. In this study, we enrolled 112 elderly patients with sepsis who were admitted to The Affiliated Hospital of Medical School, Ningbo University (Ningbo, China) for treatment between June 2015 and June 2017 as the subjects. Inclusion criteria: i) patients with diagnosis of sepsis in accordance with the diagnostic criteria of SIRS in Guidelines for Management of Severe Sepsis and Septic Shock (2012 Edition); ii) patients aged not lower than 60 years; iii) patients received no treatment before admission; and iv) patients whose family singed the written informed consent. Exclusion criteria: i) patients whose length of stay (LOS) in hospital was shorter than $24 \mathrm{~h}$; and ii) patients who were complicated with dysfunctions in liver or kidney, malignant tumors, severe hematological system diseases, or immune deficiency. Diagnostic criteria for sepsis: i) With 
confirmed infection or highly suspected infection and with more than $2 / 3$ of those items: body temperature $>38^{\circ} \mathrm{C}$ or $<36^{\circ} \mathrm{C}$; heart rate $>90$ beats $/ \mathrm{min}$; breathing $>25$ beats $/ \mathrm{min}$. ii) With more than $2 / 6$ of those biochemical indexes of inflammatory reaction: White blood cell count $>12 \times 10^{9}$ or $<4 \times 10^{9}$ or immature rate $>10 \%$, lymphocyte count decreased; serum lactate $>3 \mathrm{mmol} / \mathrm{l}$; CRP $>2$ standard deviations of normal; PCT $>2$ standard deviations of normal; serum endotoxin $>2$ standard deviations of normal; blood glucose $>7.7 \mathrm{mmol} / \mathrm{l}$ or $>110 \mathrm{mg} / \mathrm{dl}$ (no diabetes). iii) with more than $1 / 9$ of those organ dysfunction indicators: systolic blood pressure $<80 \mathrm{mmHg}$, mean arterial pressure $<70 \mathrm{mmHg}$ or adult systolic blood pressure drop $>40 \mathrm{mmHg}$; acute oliguria (urine output $<0.5 \mathrm{ml} / \mathrm{kg} / \mathrm{h}$ for more than $2 \mathrm{~h}$ ), significant edema or fluid positive balance $>20 \mathrm{ml} / \mathrm{kg}>24 \mathrm{~h}$; increases of creatinine $\geq 0.5 \mathrm{mg} / \mathrm{dl}$ (oxygenation index $<<300 \mathrm{mmHg}$ ); hyperbilirubinemia; thrombocytopenia, $<10 \times 10^{12} / 1$; bloating (reduction of bowel sounds) for more than $24 \mathrm{~h}$; coagulation abnormalities: APTT $>60 \mathrm{sec}$, INR > 1.5; Glasgow score $<14$. Patients with more than 2 items in 1 and 2 were diagnosed as sepsis. Those patients combined with 1 or more items in 3 were diagnosed as severe sepsis. Patients who did not meet the criteria of septic shock and severe sepsis were diagnosed as mild sepsis. Based on the disease condition, 112 patients were divided into two groups, i.e., the mild sepsis group $(n=68)$ and the severe sepsis group $(n=44)$. Additionally, 60 patients without sepsis who were admitted to the Intensive Care Unit (ICU) in this hospital in the same period were recruited, and those patients had no infection during hospitalization or SIRS manifestations. The study was approved by the ethics committee of The Affiliated Hospital of Medical School, Ningbo University and written informed consents were signed by the patients and/or guardians.

CK detection. We collected $5 \mathrm{ml}$ fasting venous blood in next morning after admitting to the hospital before treatment from all subjects, and samples, where ethylene diamine tetraacetic acid (EDTA) was added for anti-coagulation, were prepared for detection of levels of TNF- $\alpha$, IL-6, IL-8, PCT, ET-1 and IL-10 through enzyme-linked immunosorbent assay (ELISA) using the automatic biochemical analyzer (AU680; Beckman Coulter, Inc., Brea, CA, USA). The procedures were carried out in strict accordance with the instructions of relevant kit (Shanghai Jingkang Biological Engineering Co., Ltd., Shanghai, China).

Immunological phenotypes. The median levels of TNF- $\alpha$, IL-6, IL-8, PCT, ET-1 and IL-10 were $15.85 \mathrm{pg} / \mathrm{ml}, 33.25 \mathrm{pg} / \mathrm{ml}$, $35.85 \mathrm{pg} / \mathrm{ml}, 815.35 \mathrm{ug} / \mathrm{l}, 35.85 \mathrm{ng} / \mathrm{l}$ and $10.15 \mathrm{pg} / \mathrm{ml}$, respectively. The results above the median level were set as positive, while those below the median level were set as negative. According to the number of positive results of CKs above, we divided the status of patients into three degrees: mild inflammatory response/anti-inflammatory responses [compensatory anti-inflammatory response syndrome (CARS), 0-2 inflammatory factors with positive results/IL-10 positive], severe inflammatory responses/anti-inflammatory responses [mixed antagonist response syndrome (MARS), 0-4 inflammatory factors with positive results/IL-10 positive], and SIRS (0-6 inflammatory factors with positive results/IL-10 positive).
Prognosis. According to the worst value of patients within $24 \mathrm{~h}$ after admission, patients were scored via Acute Physiology and Chronic Health Evaluation II (APACHE II), and the survival of patients within 30 days was recorded.

Statistical analysis. Statistical analysis of all materials was performed with SPSS v18.0 (SPSS, Inc., Chicago, IL, USA). Analysis of variance with Tukey's post hoc test were performed for multiple comparisons. Measurement data were expressed as mean \pm standard deviation and compared with t-test. Qualitative data were compared with $\chi^{2}$ test. $\mathrm{P}<0.05$ was considered to indicate a statistically significant difference.

\section{Results}

General material. Comparisons of age and gender among the mild sepsis group, the severe sepsis group and the control group showed that the differences had no statistical significance $(\mathrm{P}>0.05)$, while the differences in the admission time of ICU, APACHE II scores and qSOFA scores among three groups had statistical significance $(\mathrm{P}<0.05$; Table I).

CK levels in serum of patients in varying disease conditions. In the mild and severe sepsis groups, the levels of TNF- $\alpha$, IL-6, IL-8, PCT, ET-1 and IL-10 in serum of patients were significantly higher than those in the control group $(\mathrm{P}<0.05)$, and the levels in the severe sepsis group were significantly higher than those in the mild sepsis group $(\mathrm{P}<0.05$; Table II).

CK levels in serum of alive and dead patients. Among 112 sepsis patients, there were 30 death cases (26.79\%) within $30 \mathrm{~d}$, of which the levels of TNF- $\alpha$, IL-6, IL-8, PCT, ET-1 and IL-10 were significantly higher than those in the alive patients $(\mathrm{P}<0.05$; Table III).

Immunological phenotypes. Between the mild and severe sepsis groups, as well as the survival and death patients, there were statistically significant differences in comparison of the immunological phenotypes $(\mathrm{P}<0.05$; Tables IV and V).

\section{Discussion}

Most of the sepsis is the SIRS caused by infection of pathogen, resulting in damage to the organ functions of the body. According to the American thoracic society and the Society of Critical Care Medicine, it is defined as infection and SIRS, and diagnosis should be made based on the existence of highly-suspicious infectious lesion or bacteria, while the remaining indicators are the same as those of SIRS (5). It is reported that the development of sepsis is also accompanied with the pro-inflammatory and anti-inflammatory responses, which are commonly dependent on many factors, like bacterial load, toxicity of pathogen, complications and host genetic factors $(6,7)$. In clinical research of sepsis, CK has been the major part in its pathophysiological process (8).

The results of this study showed that the levels of TNF- $\alpha$, IL-6, IL-8, PCT, ET-1 and IL-10 in serum of patients in the mild and severe sepsis groups were significantly higher than those in the control group $(\mathrm{P}<0.05)$, and the levels in the severe sepsis group were significantly higher than those in the mild 
Table I. Patient characteristics across the three groups.

\begin{tabular}{lccc}
\hline Characteristics & Control group $(\mathrm{n}=60)$ & Mild sepsis group (n=68) & Severe sepsis group (n=44) \\
\hline Gender (male/female) & $34 / 26$ & $36 / 22$ & $28 / 16$ \\
Age (years) & $66.6 \pm 3.5$ & $65.2 \pm 3.2$ & $67.6 \pm 4.5$ \\
Admission time in ICU (days) & $10.6 \pm 3.5$ & $13.5 \pm 5.6^{\mathrm{a}}$ & $18.9 \pm 6.8^{\mathrm{a}, \mathrm{b}}$ \\
APACHE II score & $10.5 \pm 3.9$ & $14.3 \pm 3.3^{\mathrm{a}}$ & $18.5 \pm 4.6^{\mathrm{a}, \mathrm{b}}$ \\
qSOFA scores & $2(1,3)$ & $4(2,5)$ & $6(5,8)$ \\
\hline
\end{tabular}

Data are presented as the mean \pm standard devaition. ${ }^{\mathrm{a}} \mathrm{P}<0.05$ vs. control group; ${ }^{\text {b }}<0.05$ vs. mild sepsis group. qSOFA, quick Sepsis Related Organ Failure Assessment; ICU, Intenstive Care Unit; APACHE, Acute Physiology And Chronic Health Evaluation.

Table II. Comparisons of the levels of TNF- $\alpha$, IL-6, -8 and -10, PCT and ET-1 in the serum of patients.

\begin{tabular}{|c|c|c|c|}
\hline Factors & Control group $(n=60)$ & Mild sepsis group $(n=68)$ & Severe sepsis group $(n=44)$ \\
\hline TNF- $\alpha(\mathrm{pg} / \mathrm{ml})$ & $10.35 \pm 2.12$ & $12.96 \pm 3.19^{\mathrm{a}}$ & $16.38 \pm 4.33^{\mathrm{a}, \mathrm{b}}$ \\
\hline IL-6 (pg/ml) & $16.35 \pm 5.35$ & $30.25 \pm 8.89^{a}$ & $39.78 \pm 12.52^{\mathrm{a}, \mathrm{b}}$ \\
\hline IL-8 (pg/ml) & $17.35 \pm 4.83$ & $29.78 \pm 6.31^{\mathrm{a}}$ & $36.15 \pm 7.22^{\mathrm{a}, \mathrm{b}}$ \\
\hline $\mathrm{PCT}(\mu \mathrm{g} / \mathrm{l})$ & $736.56 \pm 112.35$ & $1,238.75 \pm 135.73^{\mathrm{a}}$ & $1,678.96 \pm 102.56^{\mathrm{a}, \mathrm{b}}$ \\
\hline ET-1 (ng/l) & $22.26 \pm 6.35$ & $38.79 \pm 7.35^{\mathrm{a}}$ & $45.33 \pm 9.58^{\mathrm{a}, \mathrm{b}}$ \\
\hline IL-10 (pg/ml) & $4.36 \pm 1.12$ & $9.35 \pm 2.08^{\mathrm{a}}$ & $13.96 \pm 3.25^{\mathrm{a}, \mathrm{b}}$ \\
\hline
\end{tabular}

Data are presented as the mean \pm standard deviation. ${ }^{\mathrm{a}} \mathrm{P}<0.05$ vs. control group; ${ }^{\mathrm{b}} \mathrm{P}<0.05$ vs. mild sepsis group. TNF- $\alpha$, tumor necrosis factor $\alpha$; IL, interleukin; PCT, procalcitonin; ET-1, endothelin-1.

Table III. Comparisons of the TNF- $\alpha$, IL-6, -8 and -10, PCT and ET-1 levels in the serum of the surviving patients and mortalities.

\begin{tabular}{|c|c|c|c|c|}
\hline Factors & Surviving patients $(\mathrm{n}=82)$ & Mortalities $(n=30)$ & t-test & P-value \\
\hline TNF- $\alpha(\mathrm{pg} / \mathrm{m})$ & $10.36 \pm 3.12$ & $25.08 \pm 5.65$ & 3.105 & $<0.01$ \\
\hline IL-6 (pg/ml) & $26.15 \pm 4.38$ & $55.43 \pm 8.52$ & 2.963 & $<0.01$ \\
\hline IL-8 (pg/ml) & $26.35 \pm 3.59$ & $48.50 \pm 5.78$ & 3.751 & $<0.01$ \\
\hline $\mathrm{PCT}(\mu \mathrm{g} / \mathrm{l})$ & $1,302.35 \pm 159.59$ & $1,710.55 \pm 251.07$ & 3.535 & $<0.01$ \\
\hline ET-1 (ng/l) & $35.12 \pm 3.35$ & $58.41 \pm 5.33$ & 4.159 & $<0.01$ \\
\hline IL-10 (pg/m) & $8.63 \pm 1.20$ & $18.08 \pm 3.75$ & 3.251 & $<0.01$ \\
\hline
\end{tabular}

Data are presented as the mean \pm standard deviation. TNF- $\alpha$, tumor necrosis factor $\alpha$; IL, interleukin; PCT, procalcitonin; ET-1, endothelin-1.

sepsis group $(\mathrm{P}<0.05)$, suggesting that the levels of $\mathrm{TNF}-\alpha$, IL-6, IL-8, PCT, ET-1 and IL-10 are correlated with the disease condition of sepsis patients, and with the progression of sepsis, the CK level in serum will be increased. TNF- $\alpha$, IL-6, IL-8, PCT and ET-1 are the pro-inflammatory factors, and TNF- $\alpha$, a kind of small molecule protein secreted by macrophages with various biological effects, can act on a variety of cells, thereby inducing a series of inflammatory responses; under the circumstance of excessive inflammatory responses, TNF- $\alpha$ can stimulate the vascular endothelial cells to constrict, thus increasing the permeability of vessels, which will permit the massive macromolecules to pass into the interstitial fluid; thus, the intercellular adhesion molecule-1 (ICAM-1) and endothelial cell adhesion molecule-1 (ECAM-1) are excessively expressed, which can damage the microcirculation and tissues $(9,10)$. Furthermore, $\mathrm{TNF}-\alpha$ can also induce the massive release of IL- 6 and IL- 8 , thereby activating the chain reactions of inflammation, and further facilitating the progression of inflammation $(11,12)$.

IL-6 can directly activate the inflammatory cells and vascular endothelial cells, and is also toxic to them; besides, it can intensify the toxicity and inflammatory responses, which can harm the tissues and cells (13). As a key member in pro-inflammatory transmitters, IL-6 can induce the differentiation of lymphocyte $\mathrm{B}$, activate the lymphocyte $\mathrm{T}$, and act on the tissues and cells through autocrine or paracrine, thus promoting the hyperplasia of extracellular matrix, promoting cell growth and being involved in the inflammatory responses (14). The level 
Table IV. Comparisons of the immunological phenotypes between the mild and severe sepsis groups.

\begin{tabular}{|c|c|c|c|c|c|c|}
\hline Group & Cases (n) & CARS, n (\%) & MARS, n (\%) & SIRS, n (\%) & $\chi^{2}$ test & P-value \\
\hline Mild sepsis & 68 & $12(17.65)$ & 7 (10.29) & 49 (72.06) & 5.963 & 0.015 \\
\hline Severe sepsis & 44 & $9(20.45)$ & $26(59.09)$ & $9(20.45)$ & & \\
\hline
\end{tabular}

CARS, compensatory anti-inflammatory response syndrome; MARS, mixed antagonist response syndrome; SIRS, systemic inflammatory response syndrome.

Table V. Comparison of the immunological phenotypes between the surviving patients and mortalities.

\begin{tabular}{|c|c|c|c|c|c|c|}
\hline Group & Case (n) & CARS, n (\%) & MARS, n (\%) & SIRS, n (\%) & $\chi^{2}$ test & P-value \\
\hline Survivors & 82 & $18(21.95)$ & $21(25.61)$ & $43(52.44)$ & 4.358 & 0.022 \\
\hline Mortalities & 30 & $2(6.67)$ & $16(53.33)$ & $12(40.00)$ & & \\
\hline
\end{tabular}

CARS, compensatory anti-inflammatory response syndrome; MARS, mixed antagonist response syndrome; SIRS, systemic inflammatory response syndrome.

of IL-6 in serum has been confirmed to be positively correlated with the sepsis condition and its mortality rate. With the activating and chemotactic activities on neutrophils, IL-8 can induce the migration of neutrophils to the infectious site and simultaneously the massive release of inflammatory mediators. A study has indicated that in patients with severe sepsis, an acute increase in IL-8 level in the early stage usually suggests a poor prognosis (15). ET has three subtypes, ET-1, ET-2 and ET-3, in which ET-1, as the sole ET secreted by vascular endothelial cells, has a potent effect on vasoconstriction. Once the vascular endothelium is injured, the secretion of ET-1 is acutely increased, inducing the thrombosis, thereby being involved in the dysfunction of vascular endothelium, which can reflect the damage to vascular endothelial cells. It has been confirmed that damaged vascular endothelial cells are involved in the development and progression of sepsis (16). Thus, the level of ET-1 in serum can reflect the severity of sepsis to a certain degree. PCT, a kind of propeptide of PCT consisting of 116 amino acids, usually serves as an indicator for diagnosis of infection. Normally, PCT is mainly secreted by the thyroid C cells, but when it comes to the pathological status, it can also be secreted by cells of liver, kidney and muscle under the stimulation of endotoxin CKs (17). In serum of healthy people, PCT usually sustains at a low level; however, under the disease condition, massive release of PCT can be attained rapidly.

IL-10, as a major anti-inflammatory CK, mainly inhibits the immune responses to inflammation. Secreted by $\mathrm{T}$ helper 2 (TH2) cells, IL-10 can suppress the release of TNF- $\alpha$, IL- 6 and IL- 8 , thus decreasing the inflammatory responses, and terminating the progression of sepsis as well as damage to the distant organs (18). In this study, the results confirmed that the level of IL-10 in serum of sepsis patients was abnormally increased, suggesting that the immune responses were obviously inhibited; especially in patients with severe sepsis, the inhibitory effect on immune responses is more significant. Additionally, the study also confirmed that the levels of CK in serum (TNF- $\alpha$, IL-6, IL-8, PCT, ET-1 and
IL-10) were correlated with the prognosis of sepsis patients, and a high level of CK might suggest a poor prognosis.

Furthermore, in this study, we also performed the immunological phenotyping for sepsis according to the CK above. Data showed that between the mild and severe sepsis groups, as well as the survival and death patients, there were statistically significant differences in comparison of the immunological phenotypes $(\mathrm{P}<0.05)$; in the patients with mild sepsis, or those alive sepsis patients, sepsis mainly exhibited as SIRS, while in patients with severe sepsis, or those dead sepsis patients, it turns into MARS with significant disorders in immunological function, which is coincident with the results of literature. Thus, this method can efficiently evaluate the immunological status of sepsis patients, and is conducive to the clinical evaluation and stipulation of immunotherapy.

In conclusion, the levels of TNF- $\alpha$, IL-6, IL-8, PCT, ET-1 and IL-10 are correlated with the condition and prognosis of elder patients with sepsis, and the assessment system for immune level based on the levels of these indicators is conducive to the stipulation of individualized immune regulation procedure and prognostic evaluation of sepsis. However, due to the limitations in selecting $\mathrm{CK}$ and insufficient time for observation, further validation and analysis are required.

\section{Acknowledgements}

Not applicable.

\section{Funding}

No funding was received.

\section{Availability of data and materials}

The datasets used and/or analysed during the present study are available from the corresponding author upon reasonable request. 


\section{Authors' contributions}

XL and BY designed the research. XL performed the experiments and analysed the data. BY drafted, revised and finalized the manuscript. All authors read and approved the final manuscript.

\section{Ethics approval and consent to participate}

The present study was approved by the Ethics Committee of The Affiliated Hospital of Medical School, Ningbo University (Ningbo, China) and written informed consents were signed by the patients and/or guardians.

\section{Consent for publication}

Not applicable.

\section{Competing interests}

The authors declare that they have no competing interests.

\section{References}

1. Schulte W, Bernhagen J and Bucala R: Cytokines in sepsis: Potent immunoregulators and potential therapeutic targets-an updated view. Mediators Inflamm 2013: 165974, 2013.

2. Landesberg G, Levin PD, Gilon D, Goodman S, Georgieva M, Weissman C, Jaffe AS, Sprung CL and Barak V: Myocardial dysfunction in severe sepsis and septic shock: No correlation with inflammatory cytokines in real-life clinical setting. Chest 148: 93-102, 2015

3. Calzavacca P, Lankadeva YR, Bailey SR, Bailey M, Bellomo R and May CN: Effects of selective $\beta 1$-adrenoceptor blockade on cardiovascular and renal function and circulating cytokines in ovine hyperdynamic sepsis. Crit Care 18: 610, 2014.

4. BaghelK, SrivastavaRN,Chandra A,GoelSK, AgrawalJ,KazmiHR and Raj S: TNF- $\alpha$, IL- 6 , and IL-8 cytokines and their association with TNF- $\alpha-308$ G/A polymorphism and postoperative sepsis. J Gastrointest Surg 18: 1486-1494, 2014.

5. Cai G, Yan J and Qiu H: The standardization of diagnosis and treatment of severe sepsis/septic shock and its practice. Zhonghua Nei Ke Za Zhi 54: 484-485, 2015 (In Chinese).

6. Jekarl DW, Kim JY, Lee S, Kim M, Kim Y, Han K, Woo SH and Lee WJ: Diagnosis and evaluation of severity of sepsis via the use of biomarkers and profiles of 13 cytokines: a multiplex analysis. Clin Chem Lab Med 53: 575-581, 2015.
7. Ye Q, Du LZ, Shao WX and Shang SQ: Utility of cytokines to predict neonatal sepsis. Pediatr Res 81: 616-621, 2017.

8. Prashant A, Vishwanath P, Kulkarni P, Sathya Narayana P, Gowdara V, Nataraj SM and Nagaraj R: Comparative assessment of cytokines and other inflammatory markers for the early diagnosis of neonatal sepsis-A case control study. PLoS One 8: e68426, 2013.

9. Wu Y, Li C, He Y, Li Q, Wang G, Wen P, Yang W and Yang Y: Relationship between expression of microRNA and inflammatory cytokines plasma level in pediatric patients with sepsis. Zhonghua Er Ke Za Zhi 52: 28-33, 2014 (In Chinese).

10. Song ZJ, Li SS, Tong CY, Yao CL, Sun Z and Huang PZ: Change of the serum pro-and anti-inflammatory cytokines in sepsis patients. Chin J Emerg Med 17: 1191-1194, 2008 (In Chinese).

11. Han SH, Kim JS, Woo JH, Jeong SJ, Shin JS, Ahn YS and Kim JM: The effect of bortezomib on expression of inflammatory cytokines and survival in a murine sepsis model induced by cecal ligation and puncture. Yonsei Med J 56: 112-123, 2015.

12. Jin M and Khan AI: Procalcitonin: Uses in the clinical laboratory for the diagnosis of sepsis. Lab Med 41: 173-177, 2010.

13. Anand D, Ray S, Bhargava S, Das S, Garg A, Taneja S, Dhar D and Srivastava LM: Proinflammatory versus anti-inflammatory response in sepsis patients: Looking at the cytokines. Crit Care 18 (Suppl 2): P13, 2014.

14. Rey C, García-Hernández I, Concha A, Martínez-Camblor P, Botrán M, Medina A, Prieto B and López-Herce J: Pro-adrenomedullin, pro-endothelin-1, procalcitonin, C-reactive protein and mortality risk in critically ill children: A prospective study. Crit Care 17: R240, 2013.

15. Jordan I, Corniero P, Balaguer M, Ortiz J, Vila D, Velasco J, Cambra FJ and Esteban E: Adrenomedullin is a useful biomarker for the prognosis of critically ill septic children. Biomark Med 8: 1065-1072, 2014.

16. Fiorentino DF, Zlotnik A, Mosmann TR, Howard M and O'Garra A: Pillars Article: IL-10 inhibits cytokine production by activated macrophages, J. Immunol. 1991. 147: 3815-3822. J Immunol 197: 1539-1546, 2016.

17. Aisiku IP, Yamal JM, Doshi P, Benoit JS, Gopinath S, Goodman JC and Robertson CS: Plasma cytokines IL-6, IL-8, and IL-10 are associated with the development of acute respiratory distress syndrome in patients with severe traumatic brain injury. Crit Care 20: 288, 2016.

18. Jin Y, Wi HJ, Choi MH, Hong ST and Bae YM: Regulation of anti-inflammatory cytokines IL-10 and TGF- $\beta$ in mouse dendritic cells through treatment with Clonorchis sinensis crude antigen. Exp Mol Med 46: e74, 2014. International (CC BY-NC-ND 4.0) License. 